\title{
Treatment of panurethral strictures using one side dissection dorsal onlay buccal mucosal graft urethroplasty
}

\author{
Gede Wirya Diptanala Putra Duarsa' \\ Gede Wirya Kusuma Duarsa² \\ Ida Bagus Putra Pramana ${ }^{3}$ \\ Paksi Satyagraha ${ }^{4}$ \\ 'General Practitioner, Faculty of Medicine, \\ Udayana University \\ ${ }^{2}$ Department of Urology, Faculty of Medicine, \\ Udayana University, Sanglah General Academy \\ Hospital Denpasar \\ ${ }^{3}$ Department of Urology, Faculty of Medicine, \\ Udayana University, Udayana University \\ Hospital \\ ${ }^{4}$ Department of Urology, Faculty of Medicine, \\ Brawijaya University, Saiful Anwar General \\ Hospital Malang, East Java
}

\begin{abstract}
The management of panurethral stricture was still challenging and controversial. We presented a case of pan urethral strictures management by using a one-sided dissection of dorsal onlay buccal mucosal graft (BMG) urethroplasty (Kulkarni technique). A 53-years old man admitted with panurethral stricture who had previously undergone several procedures. Bipolar micturition cystourethrography procedure revealed $17 \mathrm{~cm}$ stricture length. One-sided dissection dorsal onlay buccal mucosal graft urethroplasty was performed. No drain was placed. The Foley catheter was removed four weeks after surgery, and the results of the micturition were favourable. No fistulae were found at a straight erection and meatus at a normal position. The postoperative flow rate (Qmax) was $24.9 \mathrm{ml} / \mathrm{second}$. As a conclusion Kulkarni technique urethroplasty gained good outcome for panurethral stricture in our case.
\end{abstract}

Keywords: Kulkarni technique, Urethral stricture, BXO, Lichen schlerosis

\section{Introduction}

Management of panurethral stricture has changed over time. Earlier the two stages of urethroplasty were introduced by Johanson in 1953. However, that technique relied on the genital skin to build the urethra as it may increase the risk of secondary recurrence to the integration of infected skin to the reconstructed site. ${ }^{1}$ The treatment of urethral strictures using urethral reconstruction remains a challenge and varies by the site, length, depth, and thickness of the strictures. Especially for stricture with a length of more than 2 centimetres needs higher modalities and more challenging. ${ }^{2-4}$ Panurethral stricture gives high morbidity rates and affecting patient's quality of life due to challenging management approach. A better understanding in patient care, may give important role for patient's quality of life in the future. ${ }^{1-3}$ In this study, we describe our experience with one side dissection dorsal onlay buccal mucosal graft (BMG) urethroplasty technique for repairing long anterior urethral strictures.

\section{Case Report}

A 53-year-old male was admitted due to urinary retention. The patient started to complain about urinary retention after a prolonged and several attempts for indwelling urethral catheterization.
Gede Wirya Diptanala Putra Duarsa

General Practitioner, Faculty of Medicine,

Udayana University

Jl. Tukad Musi VII/3X Panjer, Denpasar, Bali diptaduarsa@gmail.com 
Bipolar micturition cystourethrography procedure revealed stricture length over $17 \mathrm{~cm}$ (Figure 1). A suprapubic cystostomy has been placed five months for initial urinary diversion. For definitive surgery, several procedures such as meatoplasty, Direct Vision Internal Urethrotomy (DVIU) and several dilatations had been carried out. However, the patient still could not urinate properly. Then, he was prepared for a onesided dissection of dorsal onlay BMG.

The patient was evaluated for stricture etiology, length, and site using bipolar micturition cystourethrography. Previous surgical reports and other endoscopic procedures, previous surgical findings, postoperative complications, and follow-up data were collected. Then, the patient was given oral antiseptic and stop smoking for two weeks for preoperative preparation.

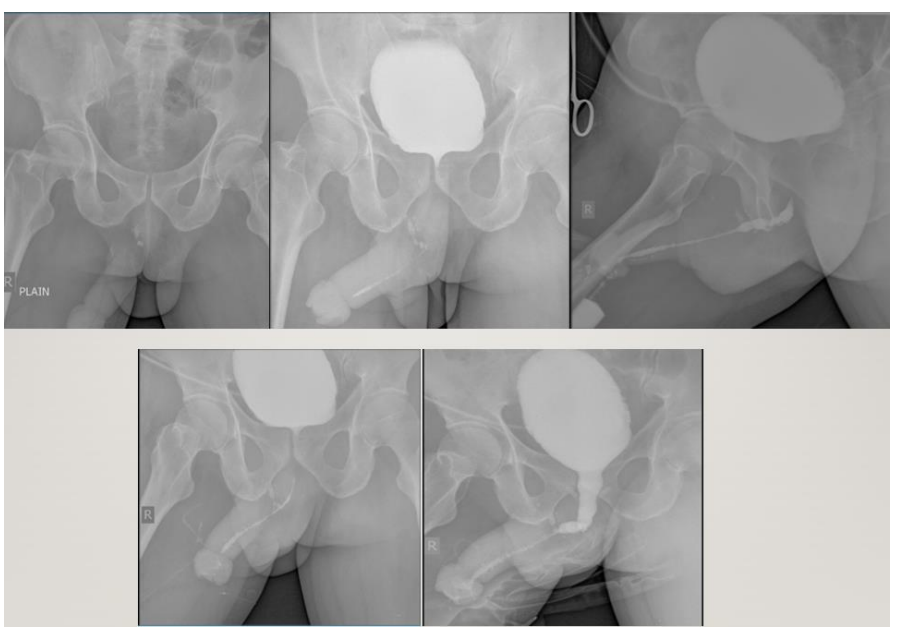

Figure 1. Urethral stricture imaging. The preoperative appearance of anterior urethral strictures with micturition cystourethrography. The figure also showed cystitis with urethritis.

General anaesthesia was selected for the surgery, and the patient was positioned in a lithotomy position. The first approach was the urethral procedure, and the second was taking the graft from the buccal mucosa. Using a perineal approach by a midline incision, penile degloving was performed to free the penis from dartos and exposed it to the corpus spongiosum of the anterior urethra. Then using a onesided dissection, the bulbar urethra was separated from corpora cavernosa to prevent the artery from facing more damages. The penile urethra was then dissected to the coronal sulcus. Further, around $17 \mathrm{~cm}$ length narrowed segment of the urethra was identified and incised in the middle part along with the stricture area (Figure 2).

The next step was harvesting the graft from the buccal mucosa; an adequate length of the graft was gathered from the inner cheek area below the Stensen's duct (Figure 3). The graft was harvested mainly from the inner cheek and extended to the lower lip if a more

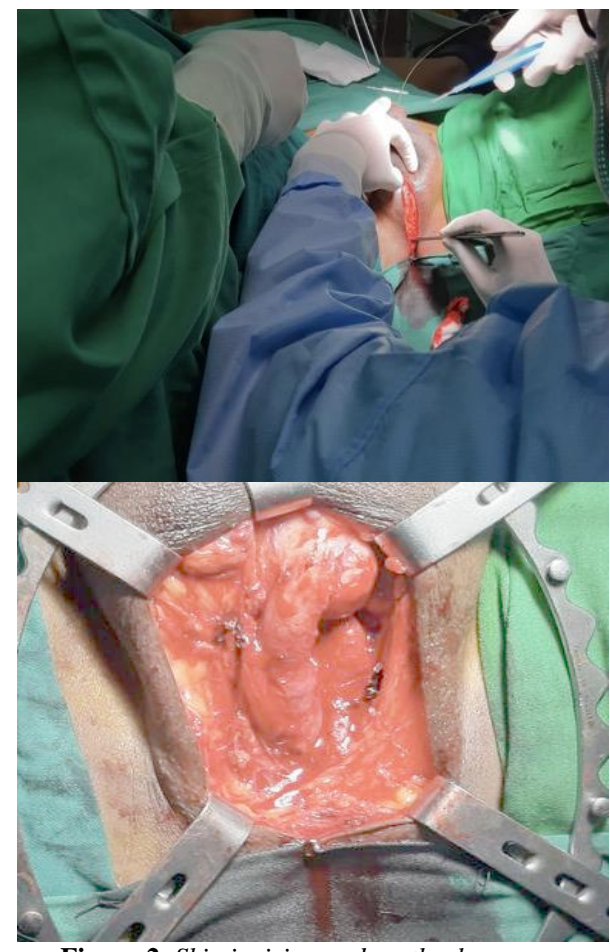

Figure 2. Skin incision and urethral exposure

extended graft was needed. By this method, a buccal mucosal graft of more than $10 \mathrm{~cm}$ can easily be harvested. Lidocaine (2\%) injection was used along with adrenaline $(1: 200,000)$ for local anaesthesia. Then the anaesthesia was used along the edges of the desired graft length to get better graft condition and preserve hemostasis. Then the graft was harvested after proper suturing of each corner were placed. Finally, defatting and tailoring procedure was done to get the appropriate graft size.

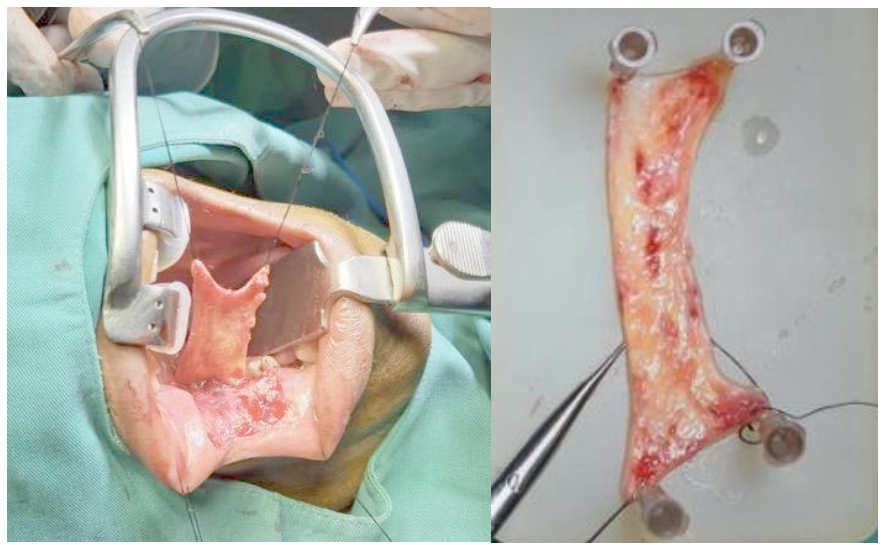

Figure 3. Buccal mucosal graft harvesting and defatted

After rotating the dissection segment about $180^{\circ}$, constricted segments of the dorsal surface were exposed and opened vertically, around $1 \mathrm{~cm}$ extended incision was made both proximal and distal of the stricture site the normal urethral lumen. Pre-harvested graft was planted by suturing and quilting it around the corpora cavernosum using 
polyglactin 5-0, reinforcing it with optimal support and minimising dead space around the planted graft. The urethral mucosa's left space was sutured to the graft using interrupted stitches of 4-0 polyglactin (Figure 4). A 16 Fr urethral catheter was inserted. Then the urethra positioned to the authentic position. Then, the remaining graft was sutured to the right space area of the urethral mucosa. Finally, the graft was covered entirely by the urethra (Figure 5).

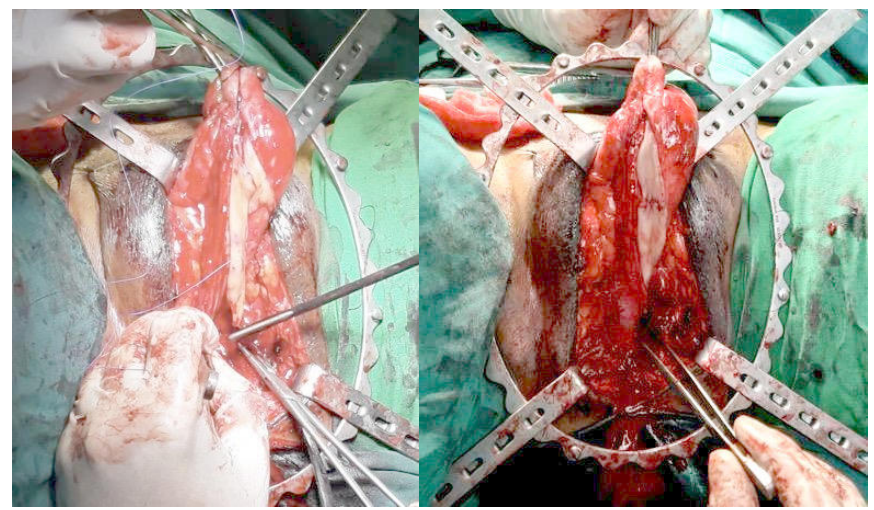

Figure 4. Suturing, splaying, and quilting the graft

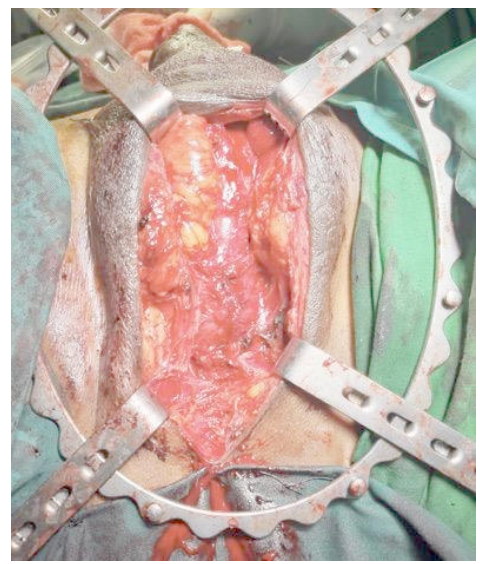

Figure 5. Wound closure

\section{Discussion}

The urethral stricture was defined as the narrowing of the calibre of the urethra due to the formation of scar tissues surrounding the urethra. ${ }^{5,6}$ Meanwhile, a panurethral stricture is a urethral stricture involving the entire urethra from the meatus until the proximal bulbar. ${ }^{4}$ Most panurethral strictures in developing countries were caused by lichen sclerosis and iatrogenic factors, such as urethral catheterisation, cystourethroscopy, transurethral resection, and previous urethral surgeries. $^{4,5}$ Other reports found trauma and sexually transmitted diseases are the most common factors. ${ }^{7}$

The urethral substitution was priorly done by using flap and grafts from the genital area. However, genital skin pedicle flaps were required advanced penoscrotal dissection for flap mobilisation to the deep perineum and associated it with penile scarring and postoperative torsion. A higher complication rate was associated with the extragenital skin flap, but better results were reported using full-thickness retro auricular skin grafts. Buccal mucosa was emerging as a choice for flap in urethral reconstruction. Buccal mucosa graft offers some advantages due to its wet environment, good vascularity, hairless, easy handling, and harvesting compared to bladder mucosa.

Moreover, it had less possibility of graft contracture and less risk of pseudodiverticulum formation. It has become popular only after 1990. Even though, Humby has already described buccal mucosa for urethral substitution in $1941.8,9$

We presented one side dorsal onlay BMG urethroplasty, and the results were favourable. No infection nor fistulae were found after operation. Meatus urethra externus is at normal position and post operative maximum flow rate (Qmax) is $24.9 \mathrm{ml} / \mathrm{second}$. In 1996, Kulkarni introduced a one-sided dissection technique using a midline incision perineal approach. This technique gives some advantages as a perineal approach to avoid incision of the penile and suture line and minimise fistula risk. Penile cosmetic outcome is excellent with no hypospadias, and no chordee was reported. Muscle and neurovascular supply around the operation site also preserved and well protected by this technique. , $^{40}$

\section{Conclusion}

Kulkarni technique urethroplasty gained good outcome for panurethral stricture in our case.

\section{Acknowledgement}

The authors would like to thank Giovani Fatrio Odo, MD, to collect the data for this study.

\section{References}

1. Pisapati VL, Paturi S, Bethu S, et al. Dorsal buccal mucosal graft urethroplasty for anterior urethral stricture by Asopa technique. Eur Urol. 2009;56(1):201-5. DOI: 10.1016/j.eururo.2008.06.002

2. Barbagli G, Guazzoni G, Lazzeri M. One-stage bulbar urethroplasty: retrospective analysis of the results in 375 patients. Eur Urol. 2008;53(4):828-33. DOI: 10.1016/j.eururo.2008.01.041

3. Kulkarni S, Barbagli G, Sansalone S, et al. One-sided anterior urethroplasty: A new dorsal onlay graft technique. BJU Int. 2009;104(8):1150-5. DOI: 10.1111/j.1464-410X.2009.08590.X

4. Kulkarni S, Kulkarni J, Surana S, et al. Management of panurethral 
stricture. The Urologic Clinics of North America. 2017;44(1):67-

75. DOI: $10.1016 /$ j.ucl.2016.08.011

5. Duarsa GWK, Subnafeu R, Oka AAG, et al. The comparable results of minimally invasive therapy versus primary anastomosis in the management of partial posterior urethral stricture. Open Access Maced $\quad J \quad$ Med Sci. 2020;8(B):435-438. DOI: 10.3889/oamjms.2020.3359

6. Reynard, J., Brewster, S. and Biers, S., n.d. Oxford handbook of urology. 3rd ed. Oxford: Oxford University Press, pp.506-551.

7. Tabassi KT, Mansourian E, Yarmohamadi A. One-stage transperineal repair of pan-urethral stricture with dorsally placed buccal mucosal grafts: results, complications, and surgical technique. Urol J. 2011;8(4):307-12.

8. Humby G. A one-stage operation for hypospadias repair. $\mathrm{Br} J$ Surg. 1941;29:84-92. DOI : 10.1002/bjs.18002911312

9. Bhargava S, Chapple CR. Buccal mucosal urethroplasty: is it the new gold standard? BJU Int. 2004;93(9):1191-3. DOI: 10.1111/j.1464-410X.2003.04860.x.

10. Kulkarni S, Joshi P, Surana S, et al. Management of panurethral stricture. African Journal of Urology. 2016;22:33-39. DOI: 10.1016/j.afju.2016.01.001 